\title{
Dressing up as Vampires: \\ Virtual vamps - negotiating female identity in cyberspace
}

MARIA MELLINS, St Mary's University College

\begin{abstract}
Dressing up as Vampires is an ethnographic audience study investigating issues of identity and the masquerade within the active female vampire fan community. Drawing on previous theoretical material by Milly Williamson (2005), Paul Hodkinson (2002) and wider methodological approaches such as Lori Kendall (1999) this paper will focus on the negotiation of female fans' identities across various online networks such as MySpace, LiveJournal, VEIN (Vampire Exchange Information Network) and Yahoo newsgroups. Its objective is to explore how identity is constructed in the virtual community via imagery (avatars \& graphics), photography and text and to what extent this online persona encroaches and impacts upon fans' 'real lives'.
\end{abstract}

Through analysis of these online networks I will suggest that female vampire fans use the Internet as an extension of their identity, and although they may construct an alternate persona within cyberspace, this usually serves as a reinforcement of their idealised 'real life' identity.

\section{KEYWORDS}

Audience, Identity, Internet, Networks.

\section{Introduction}

The recent online phenomenon of MySpace and Facebook has drawn much media attention to the role that social networks have to play within our culture. The principle objective of this research is to examine how a person's identity or identities is constructed within the virtual community, via imagery (avatars \& graphics), photography, and text. This forms part of a much broader context of Internet research that will offer new insights into the construction of on/offline identity within the virtual community.

Dressing up as Vampires is an investigation into the active female vampire fan community. It explores the negotiation of female vampire fans' identities across various online networks, focusing on a case study of one participant - MorbidFrog. MorbidFrog was selected as a case study, as she is an active member of the vampire fan group both off and online. Offline, she acts as the social secretary for one of the London based vampire fan organisations - The Vampyre Connexion. Her main duties include organising, publicising and attending events. Online, MorbidFrog is 
particularly active in generating support for Vampyre Connexion events. She channels information via various methods, such as the MySpace bulletin board, postings on her own website (http://www.morbidfrog.co.uk), frequent email updates to the Yahoo newsgroups network (thevampyreconnexion@ yahoogroups.co.uk), and the circulation of a monthly newsletter detailing a number of alternative social events relating to the vampire fan community. In selecting MorbidFrog as my case study, I am aware that her identity will be more verifiable than the majority of online vampire fans. She has and will continue to provide this research with a rich insight into subcultural practices, but a further discussion of the less verifiable members of this community will also be undertaken.

I will examine MorbidFrog's use of such online networks and consider that as Donarth (1998) states, identity is a two way process that is both established by an individual and recognised by others. Therefore, once the construction of her identity has been established, this paper will then consider the recognition of this identity by other online users, addressing issues of fan hierarchies and where MorbidFrog is positioned within the virtual community.

Finally, there will be a consideration of the wider implications of online identity and how such web networks may either reinforce or resist an individual's face-to-face identity and to what extent this online persona encroaches and impacts upon a fan's 'real life'. Through analyses of the more popular networks used by this community (MySpace, Livejournal, and to a lesser extent Yahoo newsgroups), I will suggest that female vampire fans use the Internet as an extension of their identity, and although they may construct varying presentations of the self within cyberspace, this usually serves as a reinforcement of their idealised 'real life' identity (Kendall, 1998).

\section{'Don't quote me on that', methodology and ethics}

Issues concerning research methodology and ethics are important to consider at this point. Having conducted cyberethnography for just under a year, observations have been made as to how web-users within the vampire fan community construct their identity on MySpace and Livejournal (and to a lesser extent Facebook). Because, 'nobody lives only in cyberspace' (Kendall, 1998, p.70) face-to-face participant observation was also conducted at the monthly social gatherings of the three Londonbased societies; the Vampyre Connexion, the London Vampyre Group, and the London Vampire Meetup. Via such participant observation it was possible to analyse the naturally occurring activities, interaction and sartorial practices of this community, and to essentially gain their trust.

As this paper is part of a longitudinal study a further examination into the wider aspects of fan culture will be carried out. A triangulation of methods will be employed using focus groups, questionnaires, and in-depth interviews. This research methodology will be more representational of the group as a whole and will gain a deeper insight into some of the more complex negotiations of off/online identity construction within the female vampire fan community.

Conducting cyberethnography presents researchers with certain ethical issues. Denise Carter (2005) is correct in stating that the same ethical principles apply to both 
on/offline research, 'the principle of non-maleficence, the protection of anonymity, the confidentiality of data, and the obtaining of informed consent' (2005, p.153). However, whether informed consent should be received for material that is made available within the public domain is a contentious issue. As Paccagnella (1997) suggests researchers cannot seem to agree on a common ground for ethical guidelines. Many scholars are still not explicit in their research ethics, whilst others argue over the need to obtain informed consent for information published within the public domain.

The Association of Internet Researchers (AoIR) stipulate:

The greater the acknowledged publicity of the venue, the less obligation there may be to protect individual privacy, confidentiality, right to informed consent, etc.

(Ess, 2002, p.5)

Networks such as MySpace and Livejournal both have a privacy option but the majority of profiles are publicly available and could therefore fall under the category of 'less obligation' to receive informed consent. However, this so-called 'public square' (Ibid.) model of research does not take the ephemeral nature of such networks into consideration. For instance, if researchers directly quote/publish from ephemeral web network pages such as bulletin boards or blogs, are they not denying users the power to reclaim and remove the material that they post? The AoIR states, 'you may not ethically record an otherwise ephemeral medium without consent from participants' (Ibid.).

Consequently due to the ephemeral nature of the selected online networks, informed consent was received from participants in this study. I also explicitly stated my position as a researcher on all of my online profiles to maintain a level of transparency.

\section{Online networks}

Before demonstrating the construction of identity, a brief introduction to these online networks is in order.

MySpace (http://www.myspace.com) has already proved an extremely popular medium within this community. Users can construct an online profile, select a profile tune, display photography, write blogs, and join both private communities and wider networks of likeminded individuals. They can also post forthcoming 'real life' events on the bulletin board that may be of interest to other network users (which already blurs the distinction between on/offline contexts). Most of these online profiles are open to the public and can therefore be viewed by anyone.

Similarly Livejournal (http://www.livejournal.com) is an equally popular medium amongst female vampire fans. Acting as a kind of extension to MySpace (many users post the link on their MySpace profile to redirect traffic to Livejournal) Livejournal functions as a virtual diary/blog, allowing users to write extensive entries, select from 
a variety of 'moods', and post photography detailing their daily activities. It also allows users to plan social activities and is an all round tool for keeping in touch.

In contrast Facebook (http://www.facebook.com) does not currently seem to have the same popularity amongst the active female vampire fan group. This may be due to the historical nature of the respective sites, as Facebook began as an exclusive university network where as MySpace was considered more of a music promotions network. With music being a main feature of the cultural activities of this group, it is hardly surprising that more vampire fans gravitated towards this network. However, what is perhaps more impacting on vampire fans decision to anchor towards MySpace is the technological variation of these two networks. With innovations such as theme music, custom-made pages and personalised avatars, MySpace offers users more interactive creativity and identity construction in comparison to Facebook (Gallant et $a l, 2007)$. Moreover, we can see that even from the very selection of the MySpace network (and I would argue to a similar extent their selection of Livejournal), female vampire fans are exercising their desire to express their individual identity.

Historically (fan) communities 'migrate' across different technologies to take advantage of any new developments that will assist them in building the best communities possible at that time. Therefore it is worth noting that Facebook's recent implementation of new technologies that allow users to play music, film clips and particularly the Vampire program that allows you to become a virtual vampire and score points by biting/sireing other members of the network, may result in the migration of this community to Facebook.

\section{Identity construction, 'idealised vampire image \& the body at the keyboard'}

Borrowing heuristics from Gallant et al (2007) this paper will now examine how principles of 'interactive creativity' and 'identity construction' on MySpace and Livejournal can help us to negotiate online identity, and how essentially the construction of such an online identity may involve elements of masquerading online, but these elements serve as a reinforcement of the body at the keyboard (Kendall, 1998), i.e. their face-to face persona. Moreover design elements such as graphic imagery, photography and text form an 'idealised' sense of self, rather than constructing an entirely alternate virtual persona that masquerades or quite literally dresses up as a vampire online.

Such online networks are a rich source to research as they have a level of transparency that other Usenet groups (e.g. MUDS) do not possess to the same degree. For example they have a detailed profile page that communicates personal information to virtual viewers. When viewing MorbidFrog's profile on MySpace we can immediately see an element of transparency between her cyber-identity and faceto-face identity that continues onto her use of Livejournal. We are presented with her name 'MorbidFrog', which is not an online alias as she uses both MorbidFrog and her birth name 'Cecile' in online and face-to-face environments. We also note her gender, geographical location and Date of Birth (D.O.B). We can see her physical appearance from her profile picture and various other photographs she has uploaded as a slide show. 
It is important to make the distinction here between displaying photos on the slide show as opposed to the 'my pics' section. The slide show displays photography so that all users that access this profile will inevitably see them. In contrast, the 'my pics' option assumes a level of intent from other users as they must consciously click on this option in order to access the images. Therefore, the images that MorbidFrog displays in her slideshow act as a kind of declaration of identity and are crucial to its online construction. Such images reveal a complex negotiation of identity that can be crudely separated into two categories: idealised vampire identity versus the body at the keyboard.

\section{Photography \& Imagery}

\section{Persona number1: idealised vampire identity}

This refers to a range of wish images of MorbidFrog as she poses at various gravestones both alone and with friends. She is clad in gothic apparel and appears to be presenting timeless gothic images of seduction (fig. 1), gothic elegance (fig. 2), and also horror (fig. 3). She is pictured with her permanent fangs and opts for a more 'trad goth' (Hodkinson, 2002) image over all (although her facial piercings do relate to a more modern goth/punk period). In one photograph she presents herself as an actual vampire or horror icon. Her eyes have been blacked out, her mouth is open as if she were howling, and blood drips down from her fanged mouth. MorbidFrog is essentially presenting virtual viewers with images that are synonymous with the legend of the vampire.

These images and the interactive creativity she demonstrates by her choice of black and red colour scheme, customised gothic graphics, and the use of a black and white (vampiric-in-style) profile picture, demonstrate her idealised vampire identity. However on closer inspection such an identity construction is rather paradoxical, as although MorbidFrog demonstrates a desire to be viewed as creative and individual, she is actually mirroring stereotypical goth behaviour by adhering to such gothic images.

MorbidFrog seems to be attempting to appear subversive to the world at large, but is actually rather conformist in the goth sense. Drawing on the work of Muggleton (1997), Paul Hodkinson (2002) identifies that goth culture appears to seek a degree of individuality and difference, via its sartorial trends and other fan practices. Contemporary culture is now coded by diversity whereby:

Different types of clothing, make-up, sounds and accessories float freely, available for the appropriation into a number of different individualized concoctions.

(Hodkinson, 2002, p.39)

Therefore, against this backdrop of widespread cultural diversity and a 'pick 'n' mix' of style, goth culture has been found to exaggerate its stylistic diversity and nonconformity (Hodkinson, 2002, p.39). Via MorbidFrog's creation of her idealised vampire identity she is essentially following this trend. 


\section{Persona number 2: The body at the keyboard}

The inclusion of 'real life' imagery on MorbidFrog's MySpace profile contextualises Morbid Frog's off and online experiences. For example images revealing her applying her makeup in the mirror (Fig. 4) and standing in her home (Fig. 5) reveal not only her real life context, but also her offline creativity and commitment to the culture. The creativity and precision of her eye-makeup, along with other signifiers such as the gothic art exhibition poster on the wall; crosses; Halloween pumpkins; a book case filled with dusty books; deep red roses and candles all reflect an authenticity and transparency to her off/online identity. This, and the fact that Morbid Frog's fangs, gothic dress, and makeup are a permanent fixture in her offline life suggests that although she may construct varying presentations of her identity online, the off/online identities work together to reinforce one another and unify into the idealised projection of vampire fan identity. An identity that is certainly recognisable to other members of the online vampire fan community.

\section{Text}

Text is also a major factor in identity construction. In a study carried out by Gallant $e t$ al (2007), findings suggest that the biographical elements of MySpace profiles allow users to express their identities: 'From a user's point of view, the most frequent comment by focus group participants was profiles allowed them to express their identities' (2007, p.5).

Although we can gage a certain amount from the 'About Me' section on MorbidFrog's MySpace profile, Livejournal (by her own admission) is a far more sophisticated medium for engaging with textual features such as voice and language. Morbid Frog tends to post her activities in the blogs section of MySpace and then navigate traffic to her Livejournal page, which she has used as a virtual diary for the last 3 years. For example after her recent trip to Germany for the Judgement Day festival she posts, 'I am too lazy to write another blog about it....but more details and photos are on my LJ: URL’. (MorbidFrog, 2007)

When looking at the textual features of such blogs we can make the distinction between the 'expressions given' and the 'expressions given off' and can therefore negotiate between statements that are consciously expressed, and information that is unintentionally imparted (Donarth, 1998). Considering the discourses available on both online networks a vast amount of descriptive information is expressed. For example it is evident that MorbidFrog is a single woman of French origin. Despite her heavy French accent she is a fully-fledged member of the British goth/vampire fan scene and has been in London for nearly 14 years. She participates in various subcultural activities such as the 'VC meeting, Vampire meetup, The Dev, bibliogoth, kryptorium, Not Malice, Dead and Buried, Invocation, Black Mass, Fright Club'. (Ibid.)

Her status within the vampire/goth/alternative community is high profile as we discover that she often holds talks at occult bookshops and organises events for the Vampyre Connexion and other alternative groups. She is well educated as she 
currently works as a librarian, has completed a dissertation and a postgraduate Masters, and in her spare time writes articles for the vampire fanzine 'Dark Nights':

I love anything gory, involving the black arts and supernatural. Any occult subject fascinates me. I was able to mix all those interests in my dissertation and doing all my research about occult collections.

(Ibid.)

She is also artistic, with a background in ceramics, tattooing, and painting. We also discover the origin of her name 'MorbidFrog' when she states:

My love for the pretty creatures know as grenouille and my penchant for the macabre created my nickname MorbidFrog and has been for as long as I remember.

(Ibid.)

The inclusion of her birth name and abundance of information relating to her offline life, continues the level of transparency between her body at the keyboard and online construction.

These discourses also reflect other social and cultural aspects of Morbid Frog's off/online life that are not necessarily consciously expressed. By her frequent citing of vampire-related activities and alternative lifestyle MorbidFrog appears to be reaffirming her prestigious position in the London vampire fan community. The repeated nature of her education and professional interest in the occult, on the blog sections of Livejournal/MySpace pages and on her own personal website (which holds a version of her dissertation together with her academic work history), serves to reinforce the authenticity of her interest and her dedication to the culture as a whole. Other factors such as her spelling of vampire with an 'i' (vampire) rather than the Romanian spelling with a ' $y$ ' (vampyre), her association with vampire fan groups (such as the Vampyre Connexion and the London Vampire Meetup) that are non roleplaying, non blood-drinking organisations, all assert her position as a 'fan'. MorbidFrog does not believe herself to be an actual vampire, and she does not engage with any sanguinarian/blood letting activities.

Livejournal is particularly interesting when considering the negotiation of MorbidFrog's online identity. Aside from its extensive profile page Livejournal also hosts MorbidFrog's virtual diary that she has updated daily for the last 3 years. Her journal catalogues her participation in the London goth/vampire scene. Entries include her experiences at alternate bring and buy sales, various graveyard walks, clubbing at numerous locations, and her travels further a field to various alternative festivals in Germany, Austria and Venice.

Her Venetian diary entry is of particular interest as MorbidFrog presents other network users with her idealised vampire identity. However, she is now using the online medium to express the pleasure she feels in her offline masquerade (or 'real life' fulfilment of the wish image). She states of the Venice festival: 'Venice was as beautiful as I remembered it, but on top being able to wonder the street all day long in a mask and a cape was something magical' (Ibid.) 
This continues to blur the distinction between MorbidFrog's off and online experiences and reinforces the notion that she does not create an identity online that masquerades independently from her body in cyberspace, as she is essentially describing the pleasure she feels in her real life, face-to-face fan experiences.

Therefore, MorbidFrog's off/online identities are somewhat interchangeable. The idealised vampire identity can be continued offline as well as online. She may construct multiple personas, but these identities are notable in both her online and offline experiences. She does not use networks such as MySpace and Livejournal to masquerade online and forget her 'tedious body typing at the keyboard' (Kendall, 1998, p.61). Rather, she constructs 'varying presentations of the self' (Ibid.) in both real life and the virtual world. As Kendall (1998) asserts the presentation of multiple identities was occurring well before the Internet.

\section{Identity recognition, 'Virtual vamp celebrity?'}

Having outlined the construction of identity within these virtual networks, this paper will now consider the recognition of such identity and how this recognition may impact on the virtual community.

Gallant et al (2007) discuss that such online networks are organised in a selective hierarchy:

All social systems develop structures that unite, divide and assemble people into groups, systems and networks. Selective hierarchy establishes leadership, pecking orders, and roles such as mentor, information provider, and social coordinator.

(2007, p.1).

MorbidFrog is positioned near the top of this selective hierarchy as she constructs a recognisable and coherent identity online. She holds the positions of information provider and social coordinator. Whilst it would be perilous to assume that all virtual vampires are as verifiable as MorbidFrog, her prestigious position within this community can give us rare insights into this community and gain more of an understanding of the fan hierarchies within the virtual community.

MorbidFrog appears to have constructed a recognisable virtual 'image' of herself via various online codes. The name 'MorbidFrog', the image of a frog as her cursor, the employment of a custom made black, red and grey colour schemed background on both MySpace and Livejournal all forge together as a kind of 'MorbidFrog' brand identity. Similarly her tagline on MySpace 'Everyday is Halloween' is also her signature on the Vampyre Connexion Yahoo newsgroup, which reads as if it were a commercial email signature. 'Everyday is Halloween, To know everything I get up to http://morbidfrog. livejournal. com/, http://www.myspace. com/morbidfrog'. (MorbidFrog, 2007)

Her role as information provider and social coordinator relies heavily on the recognition of her position by the rest of the group and their inherent belief in the authenticity of her information claims. Other users do invest a level of trust and belief 
in MorbidFrog that has been earned by her commitment to the position of social coordinator and the truth in the information she imparts. Via her Yahoo newsgroup emails to members of the Vampyre Connexion; events postings on the MySpace Bulletin Board; entries on the 'Friends Page' of Livejournal; Morbid Frog does more than establish her role as information provider and social coordinator, but achieves a high profile position of 'veritable celebrity' (Hodkinson, 2002 p.181) within the communities selective hierarchy.

Her position is further compounded via other users' complimentary public posting on her MySpace and Livejournal pages. For examples one network user posted their appreciation: "Thanks for re-acepting me as a friend and for keeping me informed about events. myspace is such a good place to easily find what to do in your free time' (Anon, 2007).

The amount of comments and interest that MorbidFrog's own postings receive also reflects not only her position, but also the recognition of her status by the rest of the community. As I have found with my own Livejournal account, many postings do seem to fall into the black hole of cyberspace. However the majority posted by MorbidFrog get a number of responses, and one particular review of Gaslight at the Old Vic received 13 separate responses. Similarly, the fact that alternative dress shops such as 'Frock Me', and bands such as 'The Museum of Vampires' and 'Violet Stigmata' all use the 'comments' section of her MySpace profile as an advertising forum would also suggest her page receives a certain amount of traffic.

Via MorbidFrog's case study it is possible to observe wider trends that are occurring on virtual networks and gain insights into (sub) cultural Internet use. As we have seen via the interactive nature of Morbid Frog's pages, traffic is filtered through this community from profile-to-profile (i.e. MorbidFrog's MySpace profile to her Livejournal account), to other community members' profiles, to groups pages (such as the Vampyre Connexion website), or to commercial advertising sites. In this sense the vampire fan community is not dissimilar to the goth community discussed by Paul Hodkinson in Goth.Net (2003). It is comparable to a virtual members club, where each site links to another autonomous site forming a wider 'infrastructure' (2003, p.177) or sub-network. News and events can spread much faster than they could ever hope to face-to-face and users can feel part of a virtual community.

The homogeneous nature that is emerging here, reveals that these virtual communities are serving as reinforcement of both a group and an individual's identity. Much like the face-to-face vampire fans that are bound together by membership cards, social activities, and sartorial practices the same principles seems to be emerging online, only you exchange your membership card for a login and password. As Milly Williamson suggests:

Cyberspace, with its myriad of little consensual communities, is a place where you will go in order to find confirmation and endorsement of your own identity.

(Williamson, 2000, p.249).

Further, this interconnected infrastructure of the vampire fan network also has implications on identity, as it makes masquerading online much more complex. A 
user's identity may feature on an array of different sites (MySpace, Livejournal, Facebook etc.), exposing any inconsistencies across the medium. The volume of personal information held on such profiles, and the fact that the majority of network users may have some relationship offline, combine to produce a level of online transparency.

\section{Summary: The vampire you know and the vampire you don't}

This paper has begun to investigate the negotiation of female vampire fan's identity online. As was evident with the case study of MorbidFrog, it has found that female vampire fans may construct varying presentations of the self in both face-to-face and virtual contexts but these personas act as an extension of their real life, idealised identity.

The construction of an online identity relies not only on user's ability to manipulate the imagery, photography, text and new technologies available, but also on the recognition of such a construction by the other members of the sub-network/virtual community. Although this model of approaching identity as a two way process (of firstly identity construction and then recognition), has proved productive for the sake of this paper, future research will consider that there may be something occurring inbetween these negotiations. As Judith Butler (1990) suggests, identity is not fixed there are many different negotiations.

It is now crucial to conduct further research and examine the wider offline context of this sub-cultural community in order to understand the complexities of both their identity constructions, and their interaction with other members. Cyberspace is not a 'utopian' world (Kendall, 1998, p.60), and when researching such online networks it would be fatal to ignore network users' offline relationships, as the majority of MySpace and Livejournal community members have some degree of relationship offline.

Recent online research has revealed a pattern of forging relationships online and then migrating offline. For example Milly Williamson (2005) found that online female vampire fans do not let their geographical location separate them. Williamson goes onto discuss that female vampire fans who met online also desired to have a close proximity offline (2005, p.177). As Williamson herself communicates, this is in fact in opposition to much previous online literature that states cyberspace creates new geographies for users to form relationships regardless of spatial/temporal constraints.

Emerging networks such as MySpace and Livejournal present researchers with an even more interesting negotiation of on/offline relationships, as users may be engaging in both 'friend seeking' online, and then moving their relationships into the real world, but also taking existing relationships from face-to-face settings and moving them into cyberspace. Either way these virtual communities have a vital role to play in 'enhancing sub-cultural participation' (Hodkinson, 2002, p.181).

There also needs to be a consideration of the active face-to-face female vampire fans that do not participate online. It has already been established that MorbidFrog has an elevated position within the Vampyre Connexion, a position that is constantly being 
reinforced and revalidated by the construction of her online identity. However, identity in cyberspace may not always be as verifiable as it is in the case of MorbidFrog. She may not be representational of the rest of the fan community. What about fans that do not engage in online practice? Or fans who participate online but do not have a vampiric profile, are they ostracised in any way? Conversely, what about online fans who are excluded because they do not have access to offline practices such as meetings? Fans may also use the Internet to a lesser extent or lurk online, visiting such sites but never participating themselves?

A further investigation into how these non-virtual and virtual-vamps are positioned within the wider sub-cultural community is now in order. There needs to be some consideration of quantitative analysis as well as the qualitative methods (questionnaires, focus groups, in-depth interviews) in order to investigate issues such as age. For example younger fans online practises will differ from older fans as lifestyle commitments such as children and career responsibilities will impact on the amount of time spent on the Internet. This may also have implications on a fan's online transparency, as younger fans may use multiple networks and have more of a presence online.

\section{References}

Butler, J.P. (1990) Gender Trouble: Feminism and the Subversion of Identity. New York: Routledge.

Carter, D. (2005) Living in Virtual Communities. An Ethnography of Human Relationships in Cyberspace. Information, Communication \& Society. 8(2): 148-167.

Clark, L. S. (1998) Dating on the net: teens and the rise of 'pure' relationships. In: Jones, S.G., ed. Cybersociety 2.0 Revisiting Computer-Mediated Communications and Community. Thousand Oaks: Sage, pp 159-183.

Donath, J. S. (1998) Identity and deception in the virtual community. In: M. Smith \& P. Kollock, ed. Communities in Cyberspace. London: Routledge, 1998, pp 29-59.

Etzioni, A. \& Etzioni, O. (1999) Face-to-face and Computer-Mediated Communities, A Comparative Analysis. Information Society. 15(4): 241-248.

Gallant, L., Boone M. \& Heap, A. (2007) Five Heuristics of Designing and Evaluating Web-Based Communities. First Monday. 12(3): 1-1.

Hine, C. (2000) Virtual Ethnography. London: Sage.

Hodkinson, P. (2002) Goth. Identity, Style and Subculture. Oxford: Berg.

Hodkinson, P. (2003) Net.Goth: Internet communication and (sub) cultural boundaries. In: Muggleton, D. \& Weinzierl, R., ed. The Post-Subcultures Reader. London: Berg, 2003, pp 285 - 298.

Kendall, L. (1998) Recontextualizing cyberspace: methodological considerations for online research. In: Jones, S., ed. Doing Internet Research: Critical Issues and Methods for Examining the Net. London: Sage, 1999, pp 57-74. 
Mann, C. \& Stewart, F. (2000) Internet Communication and Qualitative Research: A Handbook for Researching Online. London: Sage.

Markham, A. N. (1998) Life Online: Researching Experience in Virtual Space. Walnut Creek, CA: Altmira Press.

Markham, A. N. (2003) The Methods, Politics, and Ethics of Representation in Online Ethnography. In: Denzin, N.K. and Lincoln, Y.S., eds. The Sage Handbook of Qualitative Research (3rd ed.). Thousand Oaks, CA: Sage Publications Ltd, 2003, pp 793-820.

Paccagnella, L. (1997) Getting the seat of Your Pants Dirty: Strategies for Ethnographic Research on Virtual Communities. Journal of Computer Mediated Communications. 3(1).

Available from: http://jcmc.indiana.edu/vol3/issue1/paccagnella.html [Accessed 4 July 2007].

The Association of Internet Research (AoIR). Ess, C. (2002) Ethical DecisionMaking and Internet Research: Recommendations from the AoIR Ethics Working Committee. AoIR. Available from:

http://www.aoir.org/reports/ethics.pdf [accessed 13 June 2007].

Turkle, S. (1995) Life on Screen: Identity in the Age of the Internet. London: Phoenix.

Williamson, M. (2005) The Lure of the Vampire. London: Wallflower Press. 


\section{Appendix}

Fig. 1

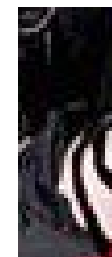

Fig. 2

Fig. 3

$B$
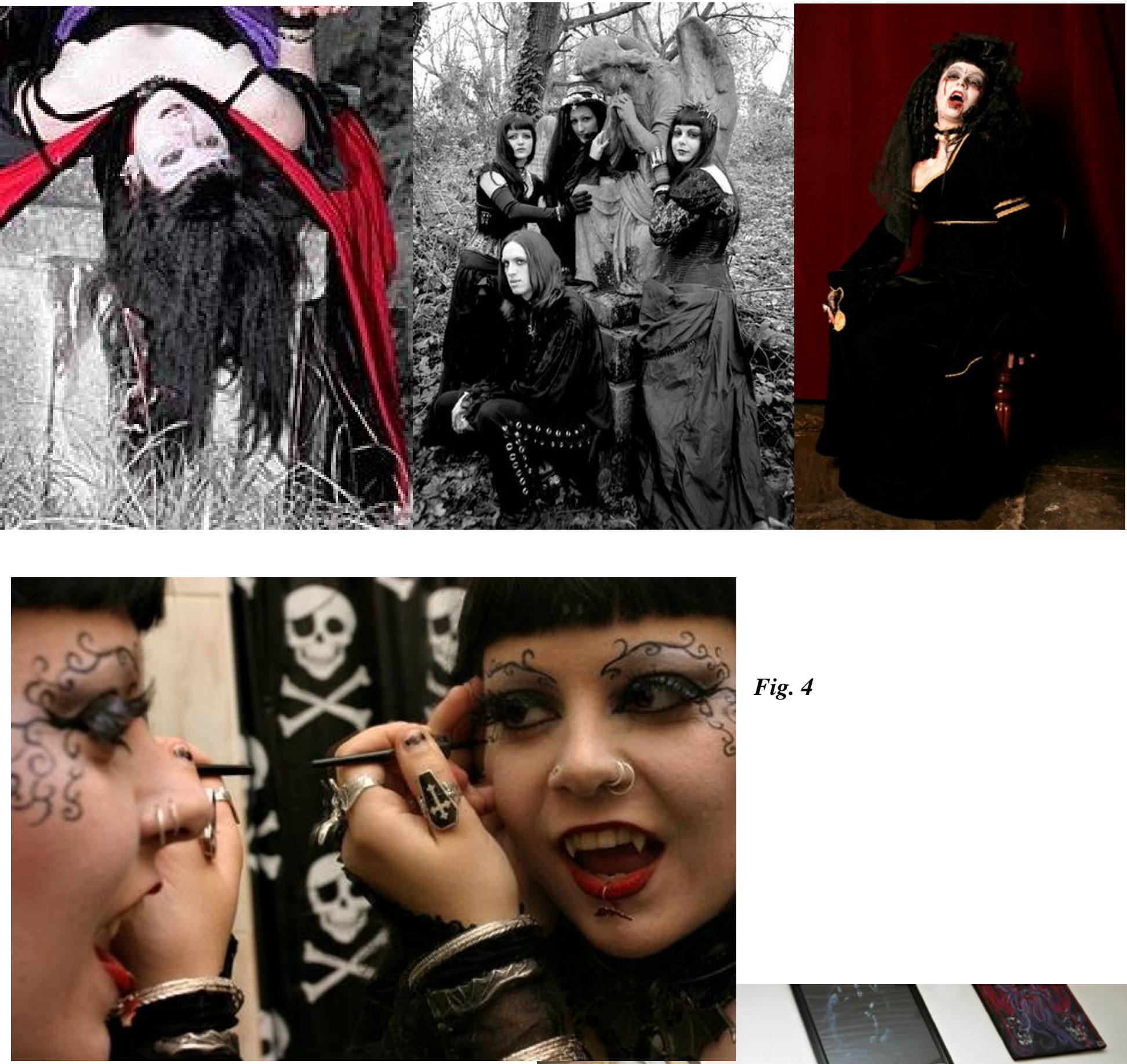

Fig. 4

Fig. 5
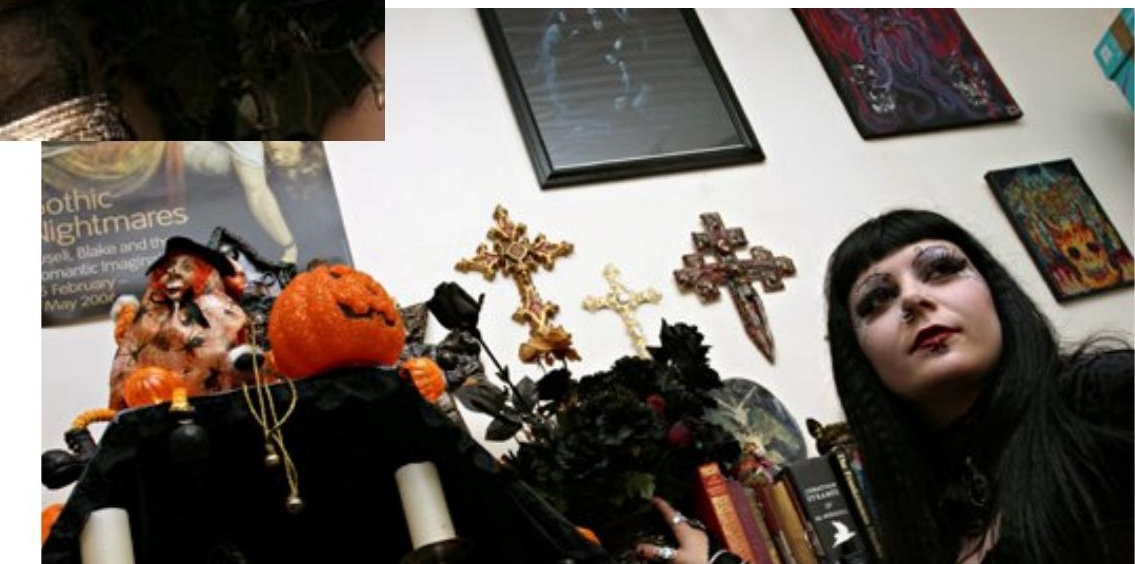